\title{
Robotic Transperitoneal Aortic Lymphadenectomy in Gynecologic Cancer: A New Robotic Surgical Technique and Review of the Literature
}

\author{
Enrico Vizza, MD, $\mathrm{PhD}^{\mathbf{1}}$, Emanuela Mancini, $\mathrm{MD}^{\mathbf{1}}$, Ermelinda Baiocco, $\mathrm{MD}^{\mathbf{1}}$, Cristina Vicenzoni, MD ${ }^{\mathbf{1}}$, \\ Lodovico Patrizi, MD, $\mathrm{PhD}^{2}$, Maria Saltari, $\mathrm{MD}^{2}$, Monica Cimino, $\mathrm{MD}^{1}$, Stefano Sindico, $\mathrm{MD}^{1}$, \\ and Giacomo Corrado, $\mathrm{MD}, \mathrm{PhD}^{3}$
}

${ }^{1}$ Gynecologic Oncologic Unit, National Cancer Institute "Regina Elena”, Rome, Italy; ${ }^{2}$ Department of Surgery, Section of Gynecology and Obstetrics, Tor Vergata University, Rome, Italy; ${ }^{3}$ Gynecologic Oncology Unit, Catholic University, Campobasso, Italy

\begin{abstract}
Background. Lymph node status is a prognostic factor for gynecologic cancer. We describe a new developing strategy for robotic transperitoneal aortic lymphadenectomy without relocating the robotic column or the patient.

Methods. Patients with histologically confirmed cervical cancer, early ovarian cancer, or endometrial carcinoma with suspected risk factors indicating aortic lymphadenectomy were eligible for the robotic transperitoneal aortic lymphadenectomy using the Da Vinci robotic system as part of the surgical treatment of gynecologic malignancies. Results. The mean operating time was $224 \mathrm{~min}$ (range 160-300 $\mathrm{min}$ ), and the mean console time for aortic lymphadenectomy was $43 \mathrm{~min}$ (range 30-75). The median hemoglobin fall was $1.3 \mathrm{~g} / \mathrm{dL}$ range $(0.8-2 \mathrm{~g} / \mathrm{dL})$, the median number of removed aortic lymph nodes was 12.5 (range 7-17), and the median length of the hospital stay was 2 days (range 1-4 days). We experienced an intraoperative complication, but no conversion to laparotomy was necessary. No patients received a blood transfusion.

Conclusions. This initial experience demonstrates the feasibility of robotic aortic lymphadenectomy with good accuracy and safety without relocating the robotic column or the patient.
\end{abstract}

Lymph node status is the most important independent prognostic factor in genital tract cancers, whereas para-aortic

(C) Society of Surgical Oncology 2012

First Received: 17 January 2012;

Published Online: 30 May 2012

G. Corrado, $\mathrm{MD}, \mathrm{PhD}$

e-mail: giacomo.corrado@alice.it lymphadenectomy is an integral part of surgical therapy in patients with ovarian, endometrial, and cervical cancer. ${ }^{1}$ The transperitoneal approach to lymph node dissection remains a technically challenging surgical intervention, especially in the case of left infrarenal nodes, the difficult removal of which is related to potential vascular complications due to the presence of large vessels, to the frequent anatomic vessel anomalies of this area, and to the limited space of the operative field. $^{2}$

Robotic surgery is a minimally invasive alternative to laparoscopy for the surgical treatment of gynecologic malignancies. The three-dimensional visualization and the seven degrees of freedom Endo Wrist (Intuitive Surgical, Sunnyvale, CA) instruments provide a marked technical improvement over traditional laparoscopy and permit a highly precise and accurate method.

One drawback of the current Da Vinci robotic system (Intuitive Surgical, Sunnyvale, CA) is the limited ability to access the entire abdominal cavity for robotic transperitoneal infrarenal aortic lymphadenectomy without relocating the robotic column.

This study was designed to evaluate a robotic transperitoneal aortic lymphadenectomy for staging of gynecologic malignancies without relocating the robotic column.

\section{MATERIALS AND METHODS}

\section{Study Design and Data Collection}

Patients with histologically confirmed early ovarian cancer defined as an ovarian tumor grossly limited to one or both ovaries with no evidence of intraperitoneal disease [stage I according to the International Federation of 
Gynecology and Obstetrics (FIGO) classification], cervical cancer with locally advanced disease, or endometrial carcinoma with suspected risk factors indicating aortic lymphadenectomy (i.e., myometrial invasion of $>50 \%$, large tumor diameter, and grade 3 or nonendometrioid pathology) were eligible for robotic transperitoneal aortic lymphadenectomy without relocating the robotic column.

Surgery was performed at the Division of Gynecologic Oncology of the National Cancer Institute "Regina Elena," Rome, Italy. All surgeries were performed on the S-system Da Vinci surgical system (Intuitive Surgical), and the surgical team consisted of the primary surgeon (E.V.), the right bedside assistant, a robotics-dedicated scrub technician, and a circulating nurse.

Clinical patient characteristics included age, body mass index, clinical stage according to FIGO, histopathologic subtype, and tumor grade. Intraoperative parameters evaluation included complications and blood loss. Blood transfusions were administrated if the hemoglobin value was $\leq 7 \mathrm{~g} / \mathrm{L}$. Postoperative parameters included short-term (within 30 days of the procedure) and long-term complications (more than 30 days after the procedure). Complications were defined as the following surgical or postoperative problems: any major vessel, nerve, or gastrointestinal injury; lymphocyst or lymphorrhea formation; and venous-thromboembolic, cardiac, pulmonary, gastrointestinal, renal/urinary tract, or urologic events. We evaluated the number and status of the pelvic lymph nodes removed, the length of the hospitalization, and the duration of median follow-up.
Approval to conduct the study was obtained independently from an internal review board. Informed consent, including clinical evaluation and robotic surgery, was obtained from all patients in accordance with local and international legislation (Declaration of Helsinki).

\section{Operative Technique}

We developed a new surgical technique for robotic aortic lymphadenectomy without relocating the robotic column. The same protocol was followed for all the operations we conducted. Patients were placed in the lithotomy position with their arms tucked at each side. After creation of a pneumoperitoneum to $12 \mathrm{mmHg}$ with a transumbilical Veress needle, a $12-\mathrm{mm}$ trocar was placed at $5-7 \mathrm{~cm}$ cranial to the umbilical. Three 8-mm trocars, specific for the Da Vinci robotic systems (Intuitive Surgical) were placed: one (arm 1) on the right side of the abdominal wall, medial and cranial to the right anterior upper iliac spine, and two on the left side of the abdominal wall, the first (arm 2) on the left lowest rib and the second (arm 3 ) medial and cranial to the left anterior upper iliac spine on the same line of the right trocar, and fastened to the robotic arms. An assistant 10-mm trocar was placed on the right side of the abdominal wall, $7-10 \mathrm{~cm}$ laterally, from the supraumbilical trocar (Fig. 1). After we obtained the Trendelenburg position $\left(30^{\circ}\right)$, the Da Vinci robotic column was positioned near the operating table between the patient's feet and docked.

The instruments were introduced: a bipolar grasper and a PK grasper on the left robotic trocars (arms 2 and 3,
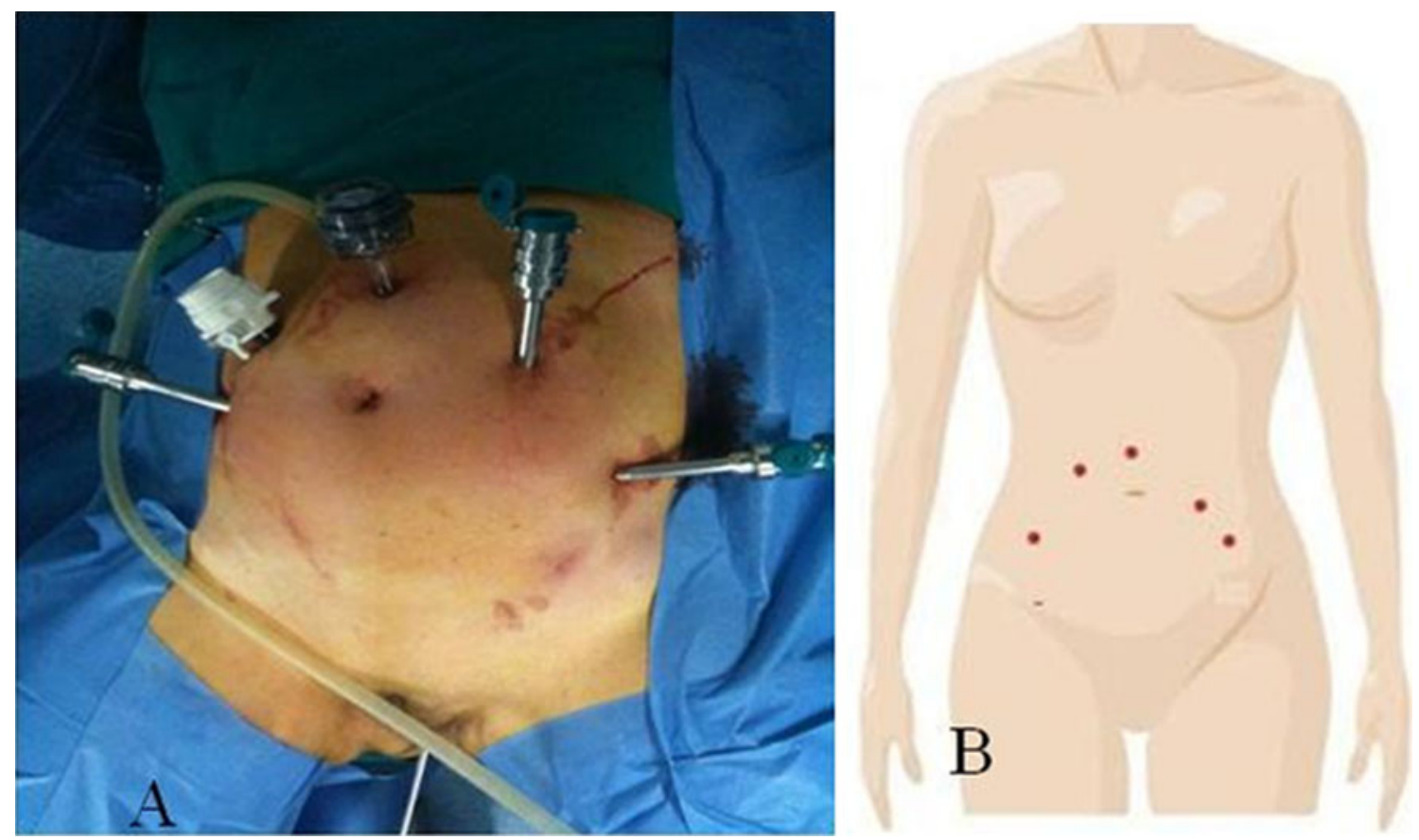

FIG. 1 Port site placement 
respectively), and a monopolar scissor on the right robotic trocar (arm 1). A $30^{\circ}$ Surgical Intuitive endoscope was used during all operations. We divided our technique for robotic aortic lymphadenectomy into four steps.

In the first step (Fig. 2), the assistant, using a laparoscopic endotract (Covidien, Mansfield, MA), packed the small bowel into the upper abdomen to improve the exposure of the common iliac artery. The peritoneum over the right common iliac artery was grasped and incised with monopolar scissors, following the right side of the aorta until the ligament of Treitz was reached, thus mobilizing the duodenum. The dissection of the retroperitoneal areolar tissue was performed in the direction of the right psoas muscle, which represented the lateral limit of our dissection. The genitofemoral nerve, ovarian vessels, and ureter are visualized. The lateral peritoneum was grasped with the ProGrasp device, and the ureter was reflected laterally.

In the second step (Fig. 3), node dissection was performed from the bifurcation of the common iliac vessels just below the left renal vein (LRV). The dissection proceeded from the medial to the lateral side, creating small pedicles of lymphatic and venous vessels that were safety
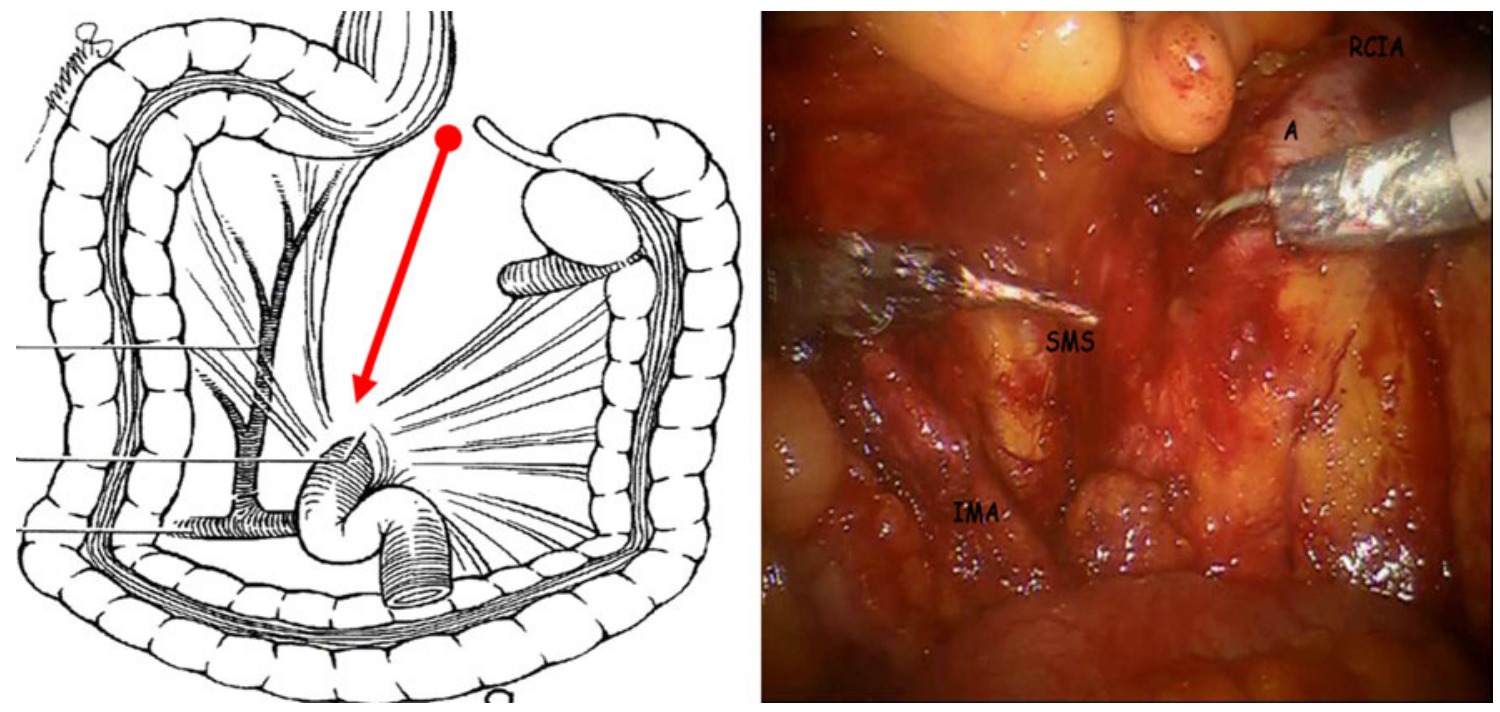

FIG. 2 Peritoneum incision. RCIA right common iliac artery, $A$ aorta, $S M S$ submesenteric space, IMA inferior mesenteric artery
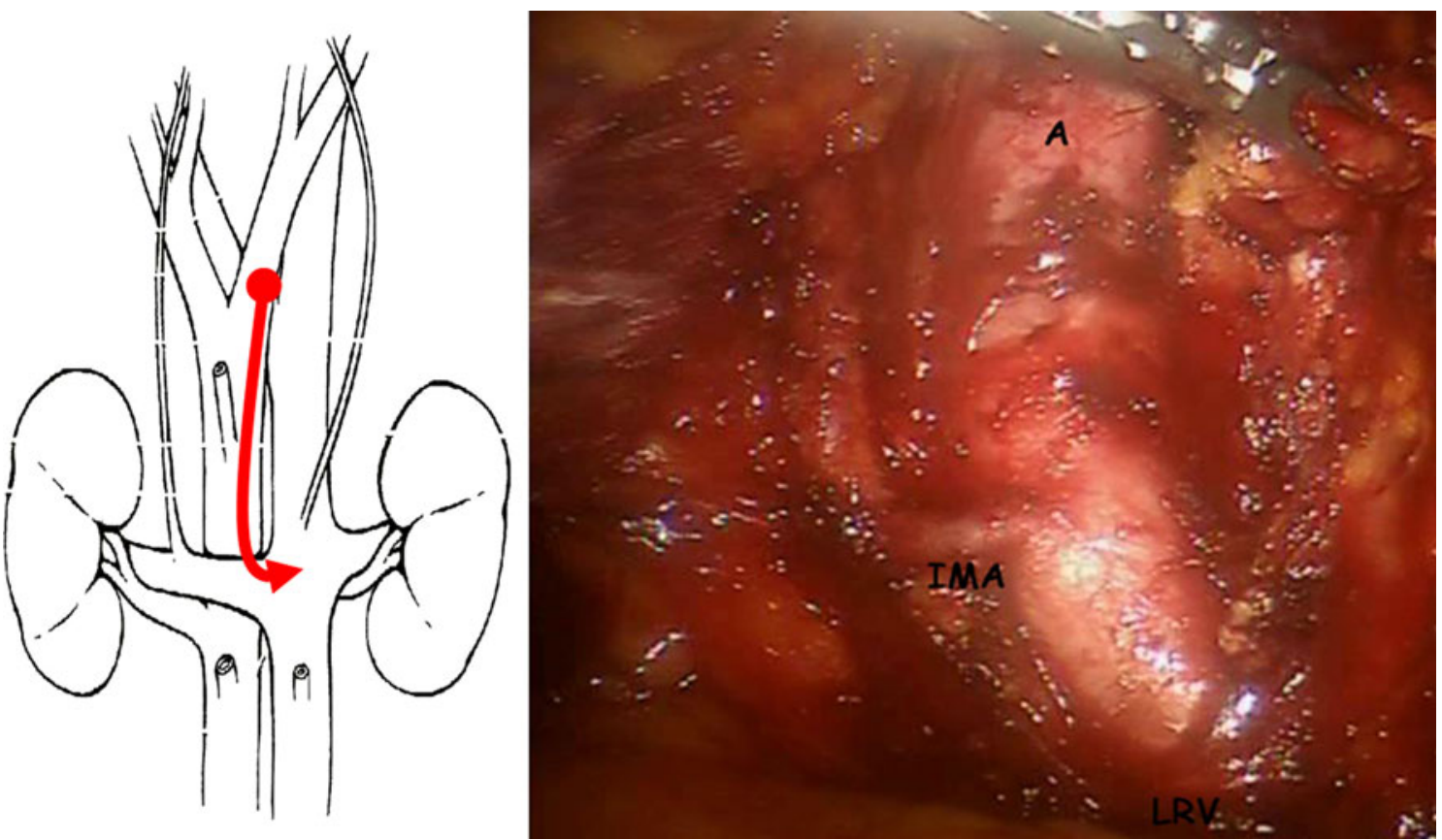

FIG. 3 Identification of the IMA and LRV. $A$ aorta 
coagulated. During this time, we visualized the inferior mesenteric artery (IMA) and LRV successively. To gain exposure to the surgical field, robotic arm 3 grasped the peritoneum reflection over the aorta and gently placed it laterally, carrying the ureter and the left ovarian vessels, which are coated to the lateral peritoneum (white line of Toldt). Once en-bloc dissection was completed, the nodes were placed into an endobag and removed through the assistant trocar.

In the third step (Fig. 4), the dissection plane was created by opening the retroperitoneal tissue under the IMA until we reached the left common iliac artery, in the direction of the left psoas muscle, which marked the lateral limit of our dissection. Then dissection was performed below the IMA to the left common vessels, and the nodes, which we placed into an endobag, were removed trough the assistant trocar. At this time, a wide field of dissection was essential for an optimal exposure, so we preferred a $30^{\circ}$ endoscope camera. An assistant created a backstop for the small bowel.

In the fourth step (Fig. 5), the dissection of the left paraaortic region above the IMA insertion into the aorta until the LRV started with a change in position of the $30^{\circ}$ camera. The camera was positioned into the assistant trocar and docked to the robotic arm while the assistant moved the instruments through the central trocar. The change of the camera's position improved the exposure of the left surgical aortic space up to the LRV (the upper limit of our
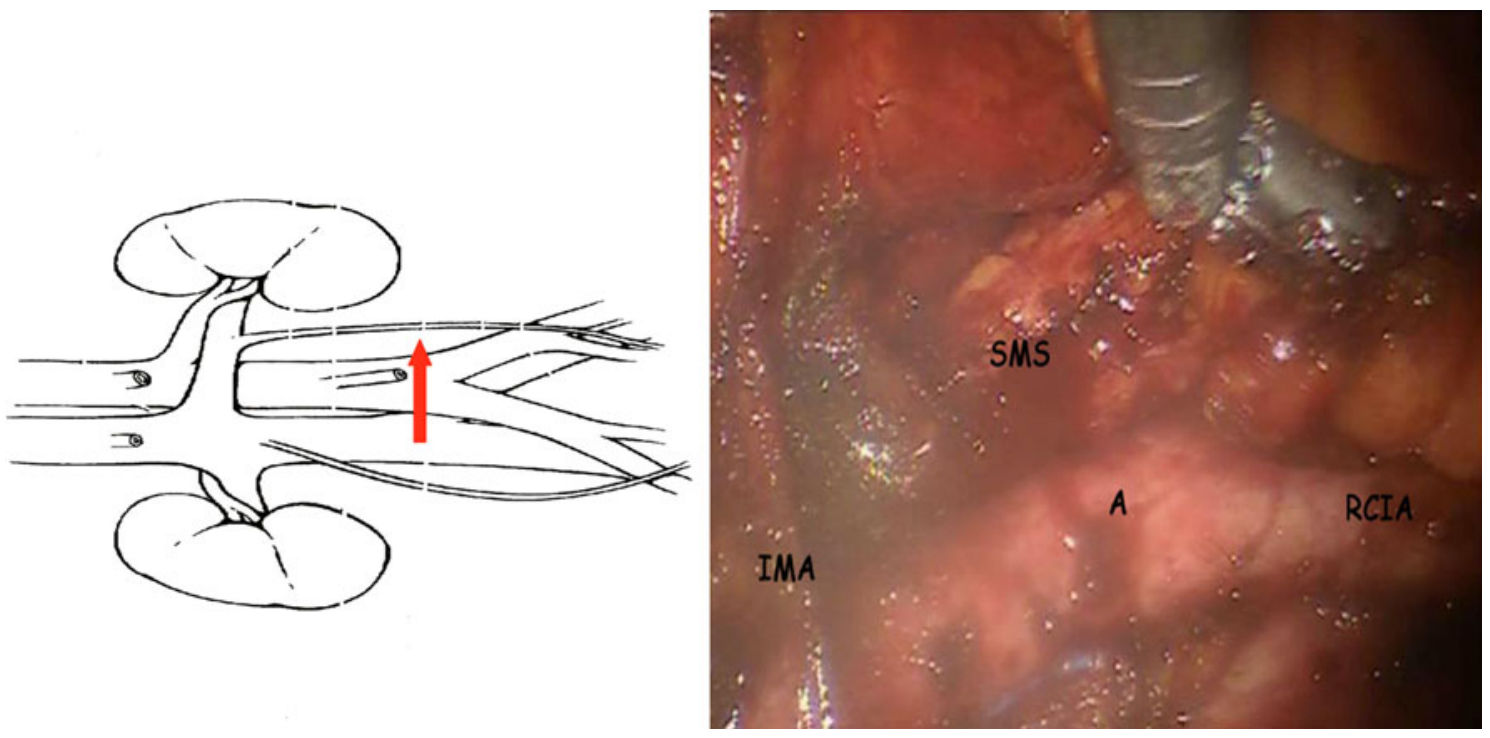

FIG. 4 SMS development. SMS submesenteric space, RCIA right common iliac artery, $A$ aorta, IMA inferior mesenteric artery
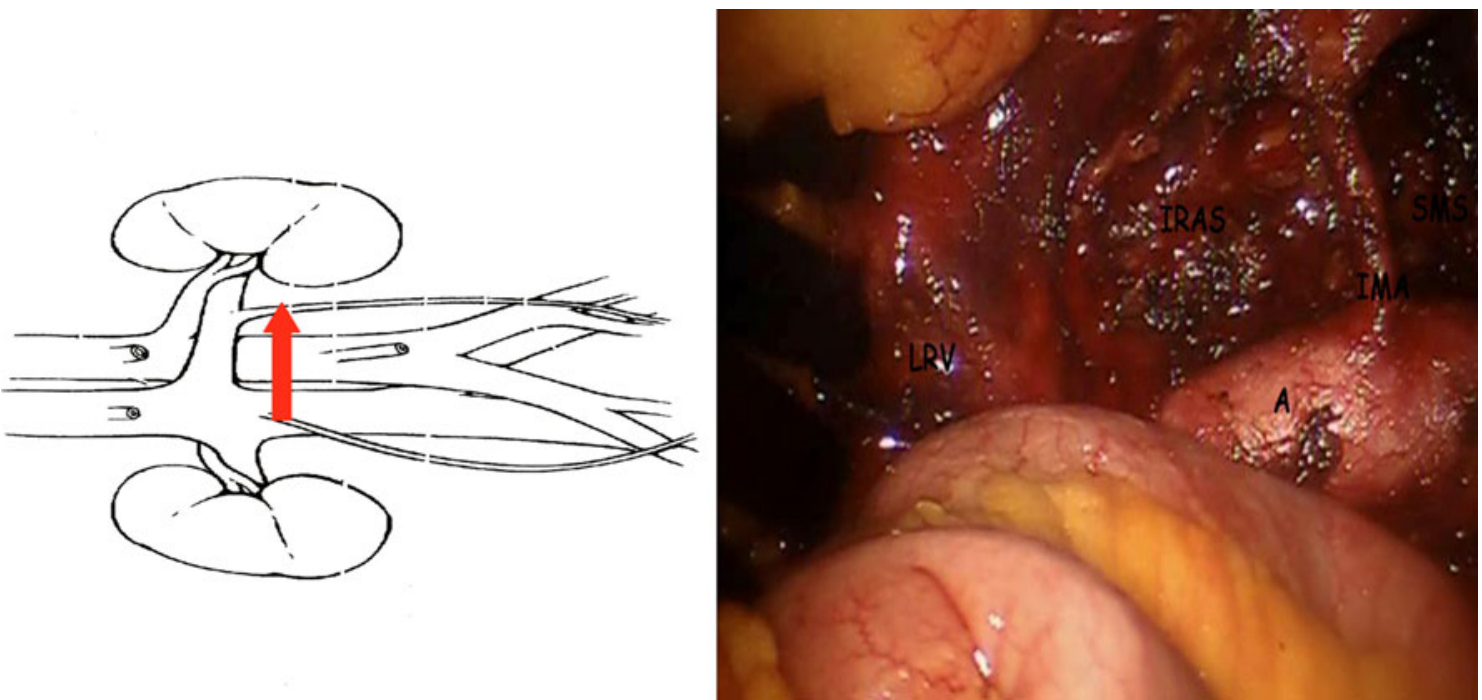

FIG. 5 IRAS development. $L R V$ left renal vein, $I R A S$ infrarenal aortic space, $S M S$ submesenteric space, $I M A$ inferior mesenteric artery, $A$ aorta 
dissection) without our having to relocate the robotic column. The retroperitoneal space was opened from IMA to the LRV in the direction of the left ovarian vessels, the lateral limit of the dissection. The lymph nodes were dissected en-bloc from IMA to the LRV, placed into an endobag, and removed via the central trocar. The lumbar vessels were not ligated, and the retrocaval and retroaortic nodes were not removed. This procedure may be interrupted prematurely if a positive node is identified at frozen section. Only suspected nodes were sent for frozen section. Retroperitoneal drainage was inserted via the $8-\mathrm{mm}$ right port cranial upper iliac spine.

\section{RESULTS}

From August 2010 to October 2011, a total of 20 patients - 2 women with locally advanced cervical cancer, 6 women with endometrial cancer, and 12 women with ovarian cancer-were treated with robotic aortic lymphadenectomy using the Da Vinci robotic system.

Thirteen patients were referred for restaging after having undergone previous procedures, as follows: 7 unilateral salpingo-oophorectomy, 3 total abdominal hysterectomy and bilateral salpingo-oophorectomy, 1 bilateral salpingooophorectomy, 1 total abdominal hysterectomy, and 1 ovarian cystectomy.

The endometrial cases were of FIGO stage IC-IIIC disease; cervical cancer, FIGO stage IIB disease; and ovarian cancer, FIGO stage IA-IIIB disease.

Additional robotic operations included 17 radical hysterectomies, 1 adnexectomy, 20 pelvic lymphadenectomies, and 6 omentectomies.

The median age of the patients was 61.3 years (range $34-73$ years); median body mass index was $27.8 \mathrm{~kg} / \mathrm{m}^{2}$ (range $21-35 \mathrm{~kg} / \mathrm{m}^{2}$ ). The mean operating time was 224 min (range 160-300 min), and the mean console time for aortic lymphadenectomy was $43 \mathrm{~min}$ (range 30-75 minutes). The time to change the camera position on the assistant trocar was only $1 \mathrm{~min}$. The mean decrease in hemoglobin measured on the first postoperative day was $1.3 \mathrm{~g} / \mathrm{dL}$ (range $0.8-2 \mathrm{~g} / \mathrm{dL}$ ), and the mean number of removed aortic lymph nodes and positive nodes was 12.5 (range 7-17) and 1 (range 0-4), respectively.

The mean follow-up was 10.4 months (range 1-21.6 months). Our most significant intraoperative complication occurred in one patient: bleeding from the right common iliac vein during pelvic lymphadenectomy. However, this was controlled robotically, and the patient's recovery was uneventful. No postoperative complication due to aortic lymphadenectomy occurred, and no conversion to laparotomy was necessary. No patients received a blood transfusion or developed a port-site metastasis. The median length of the hospital stay was 2 days (range 1-4 days), and the drainage apparatus was removed $12 \mathrm{~h}$ after surgery.

\section{DISCUSSION}

We describe a novel technique of robotic transperitoneal aortic lymphadenectomy with concomitant pelvic surgery utilizing the same patient and robotic column position.

Aortic lymphadenectomy can be performed and inframesenteric aortic nodes in most patients can be accessed and removed via robotic surgery. However, removal of the infrarenal aortic nodes up to the renal vein, and in particular the left group, in robotic surgery is often not possible, is incomplete, or is unsafe. ${ }^{3}$ The infrarenal nodes have recently received renewed interest because it is the most common site of nodal metastases in epithelial ovarian cancer and for containing positive nodes in the absence of metastases in the ipsilateral inframesenteric nodes in endometrial cancer. ${ }^{4,5}$ Moreover, para-aortic lymph node dissection in locally advanced cervical cancer may have a therapeutic benefit: no survival difference has been observed between patients with node-negative disease who received pelvic treatment alone and patients with microscopic nodal disease $(\leq 5 \mathrm{~mm})$ who are treated with definitive extended-field chemoradiotherapy. ${ }^{6}$ The procedure is even more challenging in obese patients and in patients who have undergone previous abdominal surgery, who have a distended small bowel, or who have a short small-bowel mesentery.

There is no doubt that the left infrarenal nodes are the most difficult to remove, both via laparoscopy and robotically; further, they are located in an area with potential vessel anomalies. Indicative of the surgical difficulty are the reports of the literature on robotic aortic lymphadenectomy from 2008 to 2011, where in 149 (74\%) of 201 cases the surgeon did not perform infrarenal aortic lymphadenectomy, even if the surgeon was an expert. ${ }^{3,7-12}$ Moreover, the operating time was $8-43 \mathrm{~min}$, blood loss was $50-150 \mathrm{~mL}, 1-42$ para-aortic lymph nodes were removed, and hospital stay lasted 1-13 days. The number of conversions reported was one, with intraoperative and postoperative complications of $7.5 \%$ (15 of 201) and $1.5 \%$ (3 of 201), respectively (Table 1 ).

Robot-assisted surgery has become increasingly common as a result of patient demand for minimally invasive surgery and as a result of surgeon acceptance of this new technology. Operative time, blood loss, and hospital stay seem to be equivalent when robotic and laparoscopic approaches are compared. ${ }^{13}$ These data suggest the importance of robotic surgery as a useful adjunct or valid alternative to the laparoscopic technique. Robotic surgery offers certain advantages over laparotomy and traditional 
laparoscopy, including the possibility of using threedimensional visualization and the use of instrumentation with articulating tips that allow good accuracy, precision, and safety, particularly when applied to small spaces (such as the aortic region), where the robotic apparatus enhances safety and decreases intraoperative morbidity. When surgery is performed with magnification, finer instruments can be used, and minor blood vessels can be easily identified and coagulated. Control of vessel bleeding is facilitated by the robotic instrumentation, and the maintenance of a wide field of dissection during surgery is crucial to successfully complete the surgery.

A problem, however, remains with the current robotic system in that the surgeon is limited to essentially two quadrants of the abdominopelvic cavity. This can be circumvented by patient rotation or by relocation of the robotic column. However, this is time-consuming and requires a well-trained surgical and anesthesia team. Magrina et al. $^{3}$ previously described an approach of transperitoneal infrarenal aortic lymphadenectomy involving a $180^{\circ}$ rotation of the patient when proceeding from the pelvic to aortic dissection. Although this technique has been successful, it has disadvantages, in particular the need to rotate the table, which requires coordination between the operating team and the perioperative and anesthesia personnel. Other disadvantages include the placement of several additional trocars.

This initial experience demonstrates the accuracy, safety, and feasibility of robotic aortic lymphadenectomy. The procedure described is simple and fast to perform, and it permits good exposure of the left aortic site without having to relocate the robotic column or provide an additional trocar site, thus avoiding additional time for dissection. In fact, our technique uses the same five abdominal trocars for both aortic lymphadenectomy and pelvic surgery. The number of aortic nodes and the operating time are acceptable and similar to those used during laparoscopy.

Although we described our standard protocol, it is important to individualize the port site location according to the patient's anatomy. We had no problems with tool collision, even within the limited space available. To ensure efficiency and safety, it is crucial to identify the critical retroperitoneal anatomy and the position of the small bowel and the ureter (the duodenum seems lower and the ureter more medial during robotic lymphadenectomy) before aortic node dissection in order to minimize potential intraoperative complications. We prefer to perform aortic lymphadenectomy at the end of the surgery, soon after pelvic node dissection. Changing the camera position and using the supraumbilical trocar as an assistant port during the left aortic lymphadenectomy improved the exposure of the aortic region via easier retraction of the bowel, thus allowing a better way to quickly control bleeding. To further optimize operating time and successfully complete robotic lymphadenectomy, it is important to work through time-sensitive steps, such as turning the room over, positioning the patient, docking the robot, and training the surgical team.

The disadvantages of robotic surgery, which include long operating time, lack of formal training, and higher costs, are barriers to the implementation of robotic surgery in gynecologic oncology. It is worth noting, however, that a study comparing outcomes and costs of endometrial cancer staging via traditional laparotomy, standard laparoscopy, and robotic techniques found that the most expensive approach is laparotomy as a result of longer hospitalization time. ${ }^{14}$

In conclusion, the development of our technique and the assessment of our patient series revealed that robotic aortic lymphadenectomy to the LRV after pelvic dissection (including lymphadenectomy) is feasible and safe, and it can also be carried out without robotic column rotation. A larger prospective study is now required to evaluate this procedure further.

ACKNOWLEDGMENT We thank the nursing staff for their assistance during robotic surgery, and Giuseppina Fusco for help preparing the article.

CONFLICT OF INTEREST The authors report no conflicts of interest.

\section{REFERENCES}

1. Kehoe SM, Miller DS. The role of lymphadenectomy in endometrial cancer. Clin Obstet Gynecol. 2011;54:235-44.

2. Köhler C, Tozzi R, Klemm P, et al. Laparoscopic para-aortic leftsided transperitoneal infrarenal lymphadenectomy in patients with gynecologic malignancies: technique and results. Gynecol Oncol. 2003;91:139-48.

3. Magrina JF, Long JB, Kho RM, et al. Robotic transperitoneal infrarenal aortic lymphadenectomy: technique and results. Int $J$ Gynecol Cancer. 2010;20:184-7.

4. Pereira A, Magrina JF, Rey V, et al. Pelvic and aortic lymph node metastasis in epithelial ovarian cancer. Gynecol Oncol. 2007; 105:604-8.

5. Mariani A, Dowdy SC, Cliby WA, et al. Prospective assessment of lymphatic dissemination in endometrial cancer: a paradigm shift in surgical staging. Gynecol Oncol. 2008;109:11-8.

6. Leblanc E, Narducci F, Frumovitz M, et al. Therapeutic value of pretherapeutic extraperitoneal laparoscopic staging of locally advanced cervical carcinoma. Gynecol Oncol. 2007;105:304-11.

7. Vergote I, Pouseele B, Van Gorp T, et al. Robotic retroperitoneal lower para-aortic lymphadenectomy in cervical carcinoma: first report on the technique used in 5 patients. Acta Obstet Gynecol Scand. 2008;87:783-7.

8. Fastrez M, Vandromme J, George P, et al. Robot assisted laparoscopic transperitoneal para-aortic lymphadenectomy in the management of advanced cervical carcinoma. Eur J Obstet Gynecol Reprod Biol. 2009;147:226-9. 
9. Narducci F, Lambaudie E, Houvenaeghel G, et al. Early experience of robotic-assisted laparoscopy for extraperitoneal paraaortic lymphadenectomy up to the left renal vein. Gynecol Oncol. 2009;115:172-4.

10. Holloway RW, Ahmad S, DeNardis SA, et al. Robotic-assisted laparoscopic hysterectomy and lymphadenectomy for endometrial cancer: analysis of surgical performance. Gynecol Oncol. 2009;115:447-52.

11. Seamon LG, Cohn DE, Richardson DL, et al. Robotic pelvic and aortic lymphadenectomy for endometrial cancer: the console surgeon's perspectives on surgical technique and directing the assistant. J Minim Invasive Gynecol. 2010;17:180-5.
12. Jacob KA, Zanagnolo V, Magrina JF, et al. Robotic Transperitoneal infrarenal aortic lymphadenectomy for gynecologic malignancy: a left lateral approach. J Laparoendosc Adv Surg Tech A. 2011;21:733-6.

13. Reza M, Maeso S, Blasco AJ, et al. Meta-analysis of observational studies on the safety and effectiveness of robotic gynaecological surgery. Br J Surg. 2010;97:1772-83.

14. Bell MC, Torgerson J, Seshadri-Kreaden U, et al. Comparison of outcomes and cost for endometrial cancer staging via traditional laparotomy, standard laparoscopy and robotic techniques. Gynecol Oncol. 2008;111:407-11. 